\title{
Clinical comparison of ocular and systemic findings in diagnosed cases of abusive and non-abusive head trauma
}

This article was published in the following Dove Press journal:

Clinical Ophthalmology

\author{
Linda A Morgan' \\ Samiksha Fouzdar Jain' \\ Austin Svec ${ }^{2}$ \\ Claire Svec ${ }^{2}$ \\ Suzanne B Haney' \\ Sandra Allbery' \\ Robin High ${ }^{2}$ \\ Donny W Suh ${ }^{1,2}$ \\ 'Children's Hospital and Medical \\ Center, Omaha, NE, USA; ${ }^{2}$ University \\ of Nebraska Medical Center, \\ Omaha, NE, USA
}

\begin{abstract}
Purpose: Child abuse is a leading cause of death in infants, which is often associated with abusive head trauma (AHT). The purpose of this retrospective analysis was to identify ocular and systemic findings in confirmed cases of AHT and compare them to a group of non-abusive head trauma (NAHT) patients.
\end{abstract}

Patients and methods: A retrospective chart review of 165 patients with accidental and non-accidental trauma admitted between 2013 and 2015 to Children's Hospital and Medical Center in Omaha, NE, USA, was performed. Diagnosis of AHT was made after the analysis of ocular and systemic findings by various subspecialists. The NAHT group consisted of accidental trauma, abusive trauma without significant apparent head involvement on initial evaluation and unconfirmed AHT cases.

Results: Of the 165 presenting cases, 30 patients were diagnosed with AHT and 127 were diagnosed with NAHT. Ocular findings in AHT patients were significant for retinal hemorrhages $(63 \%)$ and vitreous hemorrhages $(37 \%)$, while NAHT patients had no ocular findings $(p<0.001)$ Neuroimaging revealed subdural hemorrhages (SDHs) in 29 out of 30 AHT patients $(97 \%)$ and in 27 out of 127 NAHT patients (21\%). Seizures were present in $43 \%$ of AHT patients $(n=13)$ and only in $8 \%$ of NAHT patients $(n=10)$.

Conclusion: AHT has statistically significant findings of retinal and vitreous hemorrhages. The absence of diffuse retinal hemorrhages, however, does not preclude the AHT diagnosis as more than one-third of AHT patients lacked retinal hemorrhages. SDHs, loss of consciousness and history of seizures also have high correlation with a diagnosis of AHT.

Keywords: subdural hemorrhage, retinal hemorrhage, seizure

\section{Introduction}

Abusive head trauma (AHT) is the leading cause of infant death and long-term morbidity from injury. ${ }^{1,2}$ An estimated 30 cases in 100,000 children under the age of 1 year are diagnosed with AHT, resulting in $\sim 80$ deaths per year in the USA. ${ }^{3}$ Mortality rates from AHT are recorded as high as $25 \%$ in some cases. ${ }^{2}$ Even for those who survive, developmental delay due to brain injury and loss of vision from cortical visual impairment can be as high as $80 \%{ }^{4,5}$ Since being introduced in the 1960 s as battered infant, AHT has been known as whiplash shaken infant syndrome, inflicted traumatic brain injury and shaken baby syndrome. A definitive diagnosis of AHT can be complex and controversial, considering the social, psychological and legal ramifications. A policy statement by the American Academy of Pediatrics strongly encourages case investigations that include meticulous medical history taking, examinations and medical workups. ${ }^{6}$ Multidisciplinary specialists in radiology, ophthalmology, neurology and
Correspondence: Linda A Morgan Ophthalmology Department, Children's Hospital and Medical Center, 8400 Dodge Street, Omaha, NE 681 14, USA Tel + I 40249058 I5

Email limorgan@childrensomaha.org 
other fields may be necessary for the complete diagnosis. In addition, child protective services and law enforcement are a part of the process.

Retinal hemorrhages have been one of the hallmarks of AHT and can be severe, extend into the peripheral retina and oftentimes are described as too numerous to count. ${ }^{7,8}$ Retinal abnormalities are likely the result of rotational shearing forces between the vitreous and retina, and as many as $81 \%$ of children with AHT have bilateral hemorrhages. ${ }^{9}$ These findings are especially unique to AHT when there is no history of a traumatic accident or a coagulopathy. ${ }^{2,5}$ In addition, vitreous hemorrhages are often found in conjunction with severe retinal hemorrhages, correlating with the extreme nature of the injury. Other causes of retinal hemorrhages in children exist, including severe hematologic disease anemia, carbon monoxide poisoning, cerebral aneurysm, leukemia, infections and thrombocytopenia, which must be ruled out.

Subdural hemorrhages (SDHs) are also strongly correlated with a diagnosis of AHT when found without an associated motor vehicle accident or significant fall. ${ }^{1,10}$ Nonaccidental injury is the most common cause of significant SDHs in children under the age of 2 years. ${ }^{11}$ When associated with abuse, SDHs have a characteristic pattern of extensive diffusion in multiple areas around the brain rather than localized hemorrhages as seen in accidental blunt trauma patients. In addition to retinal and SDHs, other neurological findings are strongly associated with AHT. Among children $<1$ year of age, $95 \%$ of serious central nervous system injuries are attributed to AHT. ${ }^{1}$ One study found seizures present in $64 \%$ of AHT patients. ${ }^{12}$ The purpose of this retrospective analysis was to identify ocular and systemic findings in the cases of AHT and compare them to NAHT patients.

\section{Patients and methods}

The medical records of all children $<4$ years of age with suspected abuse admitted between 2013 and 2015 to Children's Hospital and Medical Center in Omaha, NE, USA, were retrospectively reviewed. Ethical approval for this study was granted by the institutional review board (IRB) of the University of Nebraska Medical Center, and this study complied with federal and state laws and regulations with IRB\#385-15-EP. Because it is a review of medical records, there is a waiver of parental permission (consent) and obtaining consent is impractical (45 CFR 46.116(d) and 45 CFR 164.512 (I)(2)(ii)).

A total of 165 patients were screened by hospital personnel including pediatricians, ophthalmologists, radiologists and the hospital abuse specialists, when warranted. Patients were grouped into two categories: confirmed cases of AHT and non-abusive head trauma (NAHT). All children were examined within 24 hours of their presentation to the emergency department, pediatric intensive care unit or inhospital consults. Examinations included ophthalmology examinations and computed tomography (CT) and/or magnetic resonance imaging (MRI) tests. Once a diagnosis of abuse was suspected, involvement of child protective services and law enforcement was included. A confirmed diagnosis of abuse was determined by a collection of evidence from the hospital child protection team, child protective services, law enforcement, discussions and frequent confessions from the perpetrator and, oftentimes, legal proceedings. The NAHT group was composed of accidental trauma with apparent head injury without confirmed abuse.

Retinal images were taken using Retcam III (Clarity Medical Systems, Pleasanton, CA, USA) in both groups. Retinal images and data were reviewed and recorded by a pediatric ophthalmologist. The following factors were collected and compared between the AHT and NAHT groups: head injury sequela, including fractures and SDHs; retinal and vitreous hemorrhages and associated systemic findings. Zone of retinal involvement was defined similarly to the description used for retinopathy of prematurity: zone I is the circle that has a radius twice the distance of the optic disk to the macula; zone II extends anteriorly to the nasal ora serrata; zone III is the remainder of the temporal retina.

Statistical analysis was generated with SAS/STAT software, Version 9.4 (C) 2002-2012; SAS Institute Inc. Cary, NC, USA). Variables were analyzed for statistical significance through exact chi-square testing of independence. Data results were considered significant if the $p$-value was $<0.05$.

\section{Results}

Of the 165 patients who presented with suspected abuse, 64 were specifically associated with head injuries. Of those 64 patients, 30 children had a diagnosis of AHT, and 34 children were categorized as NAHT. Male preponderance was present in both groups; AHT was 53.3\% and NAHT was $61.8 \%$ male. There were four fatalities in the AHT group, all of whom were male; there was one fatality among the NAHT group. The age of presentation of AHT patients ranged from 0.08 to 4 years with $83 \%$ of the patients under the age of 1 year. The mean age of AHT patients was 0.64 years, and the median age was 0.41 years. The age range of NAHT patients was $0.1-4$ years with a mean age of 1.6 years and a median age of 0.5 years. 
Systemic findings differed between the AHT and NAHT patients (Table 1). Loss of consciousness was more common in AHT ( $50.0 \%$ compared to $14.7 \% ; p=0.003)$. In addition, non-facial ecchymosis was a significant finding in AHT patients $(\mathrm{n}=9,30.0 \%)$ in comparison to NAHT patients $(\mathrm{n}=1$; $2.9 \% ; p=0.004)$. Seizures, lethargy and facial ecchymosis were seen more often in the AHT group. None of the patients of either group had bleeding disorders.

Ocular findings were also statistically significant in the two groups (Table 2). Retinal hemorrhages were found only in AHT $(63.3 \%$ vs $0 \% ; p<0.001)$. Of the AHT patients with retinal hemorrhages, 12 of them were found bilaterally (63.2\%) while seven had hemorrhages in one eye (36.8\%). Of the unilateral retinal hemorrhages, three were present in the right eye (42.9\%) and four were present in the left eye (56.1\%). Retinal hemorrhages were found in each layer of the retina: preretinal, intraretinal and subretinal. Specifically, intraretinal hemorrhages were found in five eyes and preretinal hemorrhages were found in seven eyes. In addition, 19 eyes had hemorrhages located in all three layers: intraretinal, subretinal and preretinal. The number of retinal hemorrhages found in an eye was documented and ranged from 5 to $>50$ with an average of 28.56 hemorrhages in

Table I Systemic findings in AHT and NAHT patients

\begin{tabular}{|c|c|c|c|}
\hline Condition & $\begin{array}{l}\text { Positive } \\
\text { findings (n) }\end{array}$ & $\%$ & $\begin{array}{l}\text { Exact } \\
\text { PV }\end{array}$ \\
\hline \multicolumn{3}{|l|}{ Seizures } & 0.19 \\
\hline NAHT & $9 / 34$ & 26.5 & \\
\hline AHT & $13 / 30$ & 43.3 & \\
\hline \multicolumn{3}{|c|}{ Loss of consciousness } & 0.003 \\
\hline NAHT & $5 / 34$ & 14.7 & \\
\hline AHT & $15 / 30$ & 50.0 & \\
\hline \multicolumn{3}{|l|}{ Ecchymosis } & 0.004 \\
\hline NAHT & $1 / 34$ & 2.9 & \\
\hline AHT & $9 / 30$ & 30.0 & \\
\hline \multicolumn{3}{|c|}{ Nausea/vomiting } & 0.27 \\
\hline NAHT & $7 / 34$ & 20.6 & \\
\hline AHT & $10 / 30$ & 33.3 & \\
\hline \multicolumn{3}{|l|}{ Lethargy } & 0.027 \\
\hline NAHT & $3 / 34$ & 8.8 & \\
\hline AHT & $10 / 30$ & 33.3 & \\
\hline \multicolumn{4}{|c|}{ Skull fractures } \\
\hline NAHT & $6 / 34$ & 17.6 & \\
\hline AHT & $7 / 30$ & 23.3 & \\
\hline \multicolumn{3}{|l|}{ Comatose } & 0.22 \\
\hline NAHT & $0 / 34$ & 0 & \\
\hline AHT & $2 / 30$ & 6.7 & \\
\hline \multicolumn{3}{|c|}{ Facial ecchymosis } & 0.018 \\
\hline NAHT & $2 / 34$ & 5.9 & \\
\hline AHT & $9 / 30$ & 30.0 & \\
\hline
\end{tabular}

Abbreviations: AHT, abusive head trauma; NAHT, non-abusive head trauma; PV, $p$-value.
Table 2 Ocular findings in AHT and NAHT patients

\begin{tabular}{|c|c|c|c|}
\hline Findings & $\begin{array}{l}\text { Positive } \\
\text { findings (n) }\end{array}$ & $\%$ & $\begin{array}{l}\text { Exact } \\
\text { PV }\end{array}$ \\
\hline \multicolumn{3}{|c|}{ Retinal hemorrhages } & $<0.001$ \\
\hline NAHT & $0 / 34$ & 0 & \\
\hline $\mathrm{AHT}$ & $19 / 30$ & 63.3 & \\
\hline \multicolumn{3}{|c|}{ Vitreous hemorrhages } & $<0.001$ \\
\hline NAHT & $0 / 34$ & 0 & \\
\hline AHT & $11 / 30$ & 36.7 & \\
\hline \multicolumn{3}{|c|}{ ONH edema } & 0.036 \\
\hline NAHT & $0 / 34$ & 0 & \\
\hline $\mathrm{AHT}$ & $2 / 30$ & 6.7 & \\
\hline
\end{tabular}

Abbreviations: AHT, abusive head trauma; NAHT, non-abusive head trauma; $\mathrm{ONH}$, optic nerve head; PV, p-value.

one eye. In addition, the size of the largest retinal hemorrhage found in one eye ranged from 0.2 to 10 disk diameters with an average of 3.17 disk diameters.

Of the 19 AHT patients with retinal hemorrhages, none had retinal hemorrhages extending into zone III. There were 16 eyes with retinal hemorrhages limited to zone I. A total of 15 eyes had retinal hemorrhages in zones I and II. While found in none of the NAHT patients, vitreous hemorrhage was found in 11 AHT patients (36.7\%), which was statistically significant $(p<0.001)$. Specifically, vitreous hemorrhages were found in 18 eyes: four unilaterally, seven bilaterally. In addition, optic nerve head edema was found in two AHT patients $(6.7 \%)$ and none of the NAHT patients. One AHT patient had bilateral optic nerve head edema, and one AHT patient had optic nerve head edema in one eye.

Parenchymal hemorrhages were noted on radiological examination in $13.3 \%$ of AHT patients, while none were in the NAHT group ( $p=0.043$; Table 3 ). SDHs had statistically significant findings in $96.7 \%$ of AHT patients and in 55.9\% of NAHT patients $(p<0.001)$. Skull fractures did not have statistical significance between the head trauma patients as they were diagnosed in $23.3 \%$ of AHT patients and $17.6 \%$ in the NAHT group $(p=0.76)$.

Table 3 Radiological findings in patients diagnosed with AHT and NAHT

\begin{tabular}{|c|c|c|c|}
\hline Findings & $\begin{array}{l}\text { Positive } \\
\text { findings (n) }\end{array}$ & $\%$ & $\begin{array}{l}\text { Exact } \\
\text { PV }\end{array}$ \\
\hline \multicolumn{3}{|c|}{ Parenchymal hemorrhages } & 0.043 \\
\hline NAHT & $0 / 34$ & 0 & \\
\hline AHT & $4 / 30$ & 13.3 & \\
\hline $\mathrm{SDHs}$ & & & $<0.001$ \\
\hline NAHT & $19 / 34$ & 55.9 & \\
\hline AHT & $29 / 30$ & 96.7 & \\
\hline
\end{tabular}

Abbreviations: AHT, abusive head trauma; NAHT, non-abusive head trauma; $\mathrm{PV}, \mathrm{p}$-value; $\mathrm{SDH}$ s, subdural hemorrhages. 


\section{Discussion}

Diagnosing child abuse carries significant weight and responsibility. False-positive allegations alter an innocent person's life with arrests and jail time, while false-negative diagnoses will leave a child in jeopardy of further danger. It is imperative for physicians to understand their role and be able to communicate their findings with skill and without bias. This retrospective analysis reviewed 165 cases of accidental and non-accidental trauma. Data between the two groups revealed statistically significant associations in confirmed cases of AHT. Retinal hemorrhages, vitreous hemorrhages and radiological findings were all strong considerations in the diagnosis. Mild or absent retinal hemorrhages, however, cannot rule out a diagnosis of AHT.

The manifestation of retinal hemorrhages carries significant weight in the diagnosis of AHT. They were present in $63.3 \%$ of AHT patients in this study compared to NAHT patients who had no retinal hemorrhages $(p<0.001)$. Other studies have shown multilayered, extensive retinal hemorrhages in up to $100 \%$ of AHT patients, in the absence of major accidental trauma or known coagulopathy., ${ }^{2,7,12,13}$ In addition, bilateral retinal hemorrhages are more common than unilateral hemorrhages in patients diagnosed with AHT, as seen in almost two-thirds of the retinal hemorrhage patients in this retrospective analysis. In Bechtel et al's ${ }^{14}$ study of abusive injury patients, $60.0 \%$ had retinal hemorrhages, of which $66.7 \%$ were bilateral. It must be noted, however, that unilateral retinal hemorrhages do not rule out a diagnosis of abuse, as they were found in seven AHT patients in the study.

Retinal hemorrhages associated with AHT are known to occur in three distinct retinal layers: preretinal, intraretinal and subretinal. While retinal hemorrhages were found in 31 AHT eyes, 19 eyes (61.3\%) had hemorrhages in all three layers. The pathophysiology of retinal hemorrhages incorporates factors such as strong vitreoretinal adhesions, the vector force involved and weaker infant blood vessels. ${ }^{5}$ Repetitive acceleration-deceleration of an infant creates a shearing between the vitreous and retina, causing traction and splitting of the retinal layers, particularly at areas of strong vitreoretinal adhesion. ${ }^{2,8,15-17}$ Research has shown that the repetition of injury will result in mechanical stress on the retina with cumulative vector force exceeding the integrity of the weak infant blood vessels, resulting in rupturing and hemorrhages. ${ }^{7,8}$

With increased vector force during abuse, severe retinal hemorrhages may give rise to vitreous hemorrhages. ${ }^{2} \mathrm{Of}$ the 31 eyes with retinal hemorrhages, 18 eyes $(58.0 \%)$ had associated vitreous hemorrhages. As they were found to be statistically significant in this study, vitreous hemorrhages were found in 11 AHT patients (36.7\%) in comparison to the NAHT patients who had no vitreous hemorrhages $(p<0.001)$. Other studies have shown between $13 \%$ and $16 \%$ of AHT patients with vitreous hemorrhages. ${ }^{12,14}$ Vitreous hemorrhages were notably higher in this study likely due to earlier detection by the abuse specialist as well as being indicative of the severity of the insult.

Other causes of retinal hemorrhages which must be ruled out in trauma cases such as these are cardiopulmonary resuscitation (CPR) and increased intracranial pressure. ${ }^{2,8,18}$ While many of the NAHT patients in this study had CPR prior to hospital admission, none of them presented with retinal hemorrhages. Studies demonstrate that hemorrhages associated with CPR may be present in the posterior pole, but they are usually few and infrequent. ${ }^{19}$ In one study of 54 patients with known CPR and no history of trauma, only one patient (2\%) had retinal hemorrhages. ${ }^{19}$ A separate, prospective study of $43 \mathrm{CPR}$ patients also found only one with retinal hemorrhages present. ${ }^{20}$ Notably, this patient had 60 minutes of CPR which resulted in small punctate hemorrhages bilaterally. ${ }^{20}$ It has been documented that the majority of CPR cases with retinal hemorrhages have concurrent coagulopathy which contributes to the bleeding. ${ }^{19,20}$

In addition, associated with retinal hemorrhages, clinically diagnosed papilledema due to increased intracranial pressure can present with a distinct pattern of retinal hemorrhages: typically intraretinal, peripapillary, splinter-like hemorrhages which are confined in or around the optic disk area, not extending to the peripheral retina. ${ }^{8}$ This pattern of hemorrhages can be distinguished from those hemorrhages found in conjunction with optic nerve changes found in AHT cases. Unlike hemorrhages, characteristic of classic papilledema and optic nerve edema found in the two AHT patients in this study were associated with more extensive retinal hemorrhages found in zones I and II as well as vitreous hemorrhages, likely secondary to the severity of the injury. Optic nerve edema associated with abuse is not a frequent occurrence. Studies have shown papilledema to be found in $<10 \%$ of AHT cases. ${ }^{8,12,14}$ In this study, only two of the patients (6.7\%) had papilledema, both with extensive retinal and vitreous hemorrhages.

While their presence is considered very important in a diagnosis, the distinct absence of retinal hemorrhages does not create a definitive diagnosis of non-abuse nor does it rule out AHT. AHT patients can present without retinal hemorrhages, as seen in 11 patients (36.7\%) in this study. 
As well, even though this study shows an absence of retinal hemorrhages in the cases of NAHT, numerous studies report a presence of minor retinal hemorrhages in $0.06 \%-10.4 \%$ after accident-related injuries. ${ }^{21,22}$

In addition to ocular findings, neurological conditions such as loss of consciousness, presence of seizures and SDHs have high correlations with AHT as found in this study. SDHs were present in 29 of the 30 AHT patients (96.7\%) in comparison to 19 of the 34 NAHT patients $(55.9 \%$, $p<0.001)$. In addition, even without the presence of any retinal hemorrhages, a remarkable 11 AHT patients were diagnosed with extensive SDHs. Similar to the vitreoretinal coup-countercoup injury resulting in retinal hemorrhages, SDHs are also secondary to the brain-cerebrospinal fluid interface..$^{23}$ The immature infant bridging veins are more susceptible to the shearing rotational forces observed in AHT, resulting in subdural and/or subarachnoid hemorrhages. ${ }^{5,24} \mathrm{In}$ contrast, NAHT patients are exposed to more translational forces such as blunt trauma, resulting in less severe epidural hemorrhages. ${ }^{5}$ Therefore, while the SDHs in AHT were found to be extensive, the SDHs in NAHT patients were found to be mild or minimal and had an association with skull fractures or benign subarachnoid fluid. ${ }^{1}$

Due to the severity of brain injury, AHT patients are also more likely to have a history of altered mental status than NAHT patients. Even though there was no statistical difference in the presence of head fractures between the two groups ( $p=0.76$ ), the degree of head injury in AHT was remarkably higher than in NAHT patients. In this study group of 30 AHT patients, $50.0 \%$ had a loss of consciousness, which was statistically significant in comparison to the loss of consciousness found in $14.7 \%$ of the NAHT patients ( $p=0.003$ ). Patients presenting with seizures or a history of seizures was not statistically significant between the two groups as they were found in $43.3 \%$ of the AHT patients and $26.5 \%$ of the NAHT $(p=0.19)$. A prospective study by Bechtel et $\mathrm{al}^{14}$ found $53 \%$ of the AHT patients to have seizures and 53\% with altered mental status. Encountering AHT patients with seizures must be compared to those patients with simple seizure disorders who do not typically present with retinal hemorrhages at all. ${ }^{25}$

A diagnosis of AHT or NAHT is obviously multifactorial and must be considered with caution. To positively diagnose a case of AHT, a combination of clinical features must be analyzed. As seen in this study, the presence or absence of retinal hemorrhages alone cannot produce a diagnosis of AHT without also analyzing any associated neurological data. In fact, the severity of brain injury often correlates highly with the severity of retinal hemorrhages. Maguire et al's large-scale study found the probability of AHT diagnosis for a child presenting with apnea and retinal hemorrhages to be $90 \% .{ }^{26}$ In addition, if a child had seizures and retinal hemorrhages, the probability of AHT was $\sim 87 \% .^{26}$

Limitations in this study include its retrospective nature, no multivariate analysis and the small sample size. In addition, retrospective nature of the analysis could have led to bias due to looking for already-known associations with AHT. In addition, these findings may be skewed, because this hospital is a level II trauma center thus the number of cases presenting with critical head trauma involving retinal hemorrhage may be limited.

\section{Conclusion}

The presence of retinal and SDHs and a history of loss of consciousness are significant risk factors for AHT. Even in unilateral or absent retinal hemorrhage cases, one should be thorough in evaluating for a potential of abuse, especially when those cases are found to occur in conjunction with SDHs. Correlating a patient's social and medical history with radiological findings are important considerations when making a diagnosis. While these findings support published literature, a large-scale prospective study is needed to collect more extensive clinical data while using a multivariate analysis.

\section{Disclosure}

The authors report no conflicts of interest in this work.

\section{References}

1. Hedlund GL. Subdural hemorrhage in abusive head trauma: imaging challenges and controversies. J Am Osteopath Coll Radiol. 2012;1:23-30.

2. Binenbaum G, Mirza-George N, Christian CW, Forbes BJ. Odds of abuse associated with retinal hemorrhages in children suspected of child abuse. J AAPOS. 2009;13(3):268-272.

3. American Academy of Pediatrics [hompage on the Internet]. Abusive Head Trauma (Shaken Baby Syndrome). American Academy of Pediatrics. Available from: http://Aap.org. Accessed June 4, 2018.

4. Barlow KM, Thomson E, Johnson D, Minns RA. Late neurologic and cognitive sequelae of inflicted traumatic brain injury in infancy. Pediatrics. 2005;116(2):e174-e185.

5. Duhaime AC, Alario AJ, Lewander WJ, et al. Head injury in very young children: mechanisms, injury types, and ophthalmologic findings in 100 hospitalized patients younger than 2 years of age. Pediatrics. 1992; 90(2 pt 1):179-185.

6. Christian CW, Block R; Committee on Child Abuse and Neglect; American Academy of Pediatrics. Abusive head trauma in infants and children. Pediatrics. 2009;123(5):1409-1411.

7. Levin AV, Cordovez JA, Leiby BE, Pequignot E, Tandon A. Retinal hemorrhage in abusive head trauma: finding a common language. Trans Am Ophthalmol Soc. 2014;112:1-10.

8. Levin AV. Retinal hemorrhage in abusive head trauma. Pediatrics. 2010; 126(5):961-970. 
9. Hoskote A, Richards P, Anslow P, McShane T. Subdural hematoma and non-accidental head injury in children. Childs Nerv Syst. 2002; 18(6-7):311-317.

10. Maguire SA, Watts PO, Shaw AD, et al. Retinal haemorrhages and related findings in abusive and non-abusive head trauma: a systematic review. Eye (Lond). 2013;27(1):28-36.

11. Tzioumi D, Oates RK. Subdural hematomas in children under 2 years. accidental or inflicted? A 10-year experience. Child Abuse Negl. 1998; 22(11):1105-1112.

12. Kivlin JD. A 12-year ophthalmologic experience with the shaken baby syndrome at a regional children's hospital. Trans Am Ophthalmol Soc. 1999;97:545-581.

13. Kivlin J, Simons K, Lazoritz S, Ruttum M. Shaken baby syndrome. Ophthalmology. 2000;107:1246-1254.

14. Bechtel K, Stoessel K, Leventhal JM, et al. Characteristics that distinguish accidental from abusive injury in hospitalized young children with head trauma. Pediatrics. 2004;114(1):165-168.

15. Thanos A, Yonekawa Y, Yomogida K, et al. Retinal findings in abusive head trauma. Retina Today. 2016(3):57-59.

16. Shiau T, Levin AV. Retinal hemorrhages in children: the role of intracranial pressure. Arch Pediatr Adolesc Med. 2012;166(7):623-628.

17. Nadarasa J, Deck C, Meyer F, et al. Update on injury mechanisms in abusive head trauma-shaken baby syndrome. Pediatr Radiol. 2014; 44(Suppl 4):S565-S570.
18. Morad Y, Kim YM, Armstrong DC, Huyer D, Mian M, Levin AV. Correlation between retinal abnormalities and intracranial abnormalities in the shaken baby syndrome. Am J Ophthalmol. 2002;134(3): 354-359.

19. Kanter RK. Retinal hemorrhage after cardiopulmonary resuscitation or child abuse. J Pediatr. 1986;108(3):430-432.

20. Odom A, Christ E, Kerr N, et al. Prevalence of retinal hemorrhages in pediatric patients after in-hospital cardiopulmonary resuscitation: a prospective study. Pediatrics. 1997;99(6):E3.

21. Aoki N, Masuzawa $\mathrm{H}$. Infantile acute subdural hematoma: clinical analysis of 26 cases. J Neurosurg. 1984;61(2):273-280.

22. Ikeda A, Sato O, Tsugane R, et al. Infantile acute subdural hematoma. Childs Nerv Syst. 1987;3(1):19-22.

23. Bandak FA. Shaken baby syndrome: a biomechanics analysis of injury mechanisms. Forensic Sci Int. 2005;151(1):71-79.

24. Feldman KW, Bethel R, Shugerman RP, Grossman DC, Grady MS, Ellenbogen RG. The cause of infant and toddler subdural hemorrhage: a prospective study. Pediatrics. 2001;108(3):636-646.

25. Watts P; Child Maltreatment Guideline Working Party of Royal College of Ophthalmologists UK. Abusive head trauma and the eye in infancy. Eye (Lond). 2013;27(10):1227-1229.

26. Maguire SA, Kemp AM, Lumb RC, Farewell DM. Estimating the probability of abusive head trauma: a pooled analysis. Pediatrics. 2011; 128(3):e550-e564.
Clinical Ophthalmology

\section{Publish your work in this journal}

Clinical Ophthalmology is an international, peer-reviewed journal covering all subspecialties within ophthalmology. Key topics include: Optometry; Visual science; Pharmacology and drug therapy in eye diseases; Basic Sciences; Primary and Secondary eye care; Patient Safety and Quality of Care Improvements. This journal is indexed on

\section{Dovepress}

PubMed Central and CAS, and is the official journal of The Society of Clinical Ophthalmology (SCO). The manuscript management system is completely online and includes a very quick and fair peer-review system, which is all easy to use. Visit http://www.dovepress.com/ testimonials.php to read real quotes from published authors. 